\title{
Illumination Invariant Moving Object Detection
}

\author{
Chirag I. Patel and Ripal Patel
}

\begin{abstract}
The main objective of this paper is to design a moving object detection model which can handle quick changes in illumination conditions. Two approaches were used for this purpose. First approach divide distortion in an image as color and brightness distortion then pixel having color distortion above a certain threshold value are classified as foreground pixels and rest as background. Second approach combines GMM with the fact that edges and gradient information remains invariant during illumination change. First model gives appreciable results while second model doesn't show much improvement over GMM.
\end{abstract}

Index Terms - Video processing, gaussian mixture model, color and brightness distortion, object detection.

\section{INTRODUCTION}

Automatic detection and recognition of objects is of prime importance for security systems and surveillance applications. Typically common approach of discriminating objects from background is finding moving object. Idea is to subtract current image from a reference background image. There are lot of Moving object detection techniques but very few of them can cope with quick illumination changes. However noisy background detection creates problems in further stages of object detection so any good surveillance system needs to have a background detection/moving object detection model to cope with problems like lightning conditions, shadows and different types of occlusions. There are lot of existing moving object detection models which can handle long term illumination changes[1] presents a method for illumination independent moving object detection using two views while[2] and [3] can handle shadows by detecting shadows as background object. In [4] moving object is detected by Gaussian mixture model but there are still the crisis of illumination changes.

\section{METHODOLOGY}

\section{A. Image Dataset}

Data set was taken from PETS2001 image dataset [5]. From this dataset image having no foreground objects were taken in training set and others were used as test data. In training set images were taken so that system can learn possible color changes in the background. Images in testing dataset have very different illumination condition as

Manuscript received October 9, 2012; revised November 19, 2012.

Chirag I Patel is with Computer Science Department, Nirma Institute of Technology, Ahmedabad, India (e-mail: chirag453@gmail.com)

Ripal Patel is with Electronics \& Commu. Department, Dharmsinh Desai institute of technology, Nadiad, India (email:ripalpate1315@gmail.com) compare to images in training dataset .Training image is shown in Fig. 1.

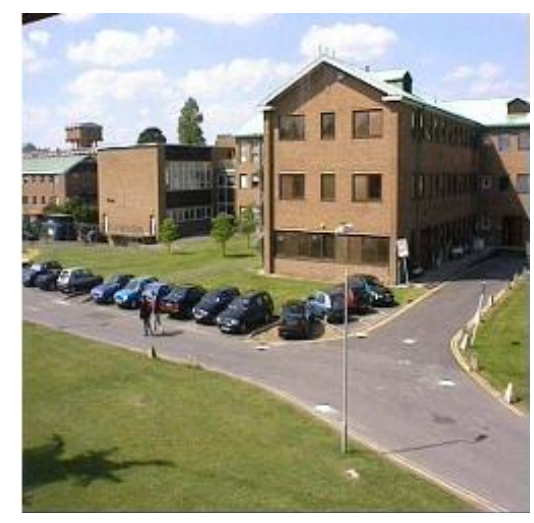

Fig. 1. Training image from PETS 2001 dataset

\section{B. Color Model}

Idea is that distortion in image from background image can be divided as sum of two distortion i.e. color and brightness distortion. Brightness will change if illuminating condition varies, but color distortion is independent up to some extent. Similar idea of dividing distortion in two parts is used in [3] shadow detection. Let

$$
E(i)=E\left(r_{i}\right) E\left(g_{i}\right) E\left(b_{i}\right)
$$

is expected color at $i^{\text {th }}$ pixel in the background image and

$$
I(i)=I\left(r_{i}\right) I\left(g_{i}\right) I\left(b_{i}\right)
$$

is at $i^{\text {th }}$ pixel value of the current image then brightness distortion $(\alpha)$ is defined as the value of $\alpha$ for which

$$
f(\alpha)=(I(i)-\alpha E(i))^{2}
$$

is minimum and now once a is known we can find out $\mathrm{CD}$ (i) as

$$
C D(i)=\bmod \left(I(i)-\alpha^{*} E(i)\right)
$$

Fig. 2 is defined color and brightness distortion in RGB space.

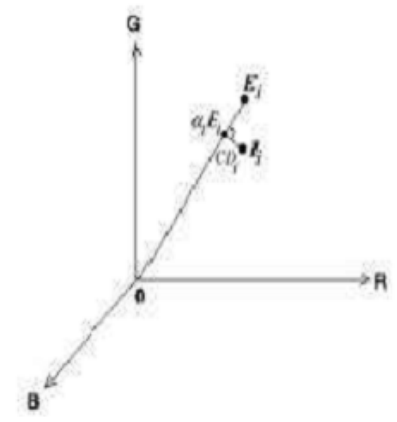

Fig. 2. Color and brightness distortion in RGB Space 


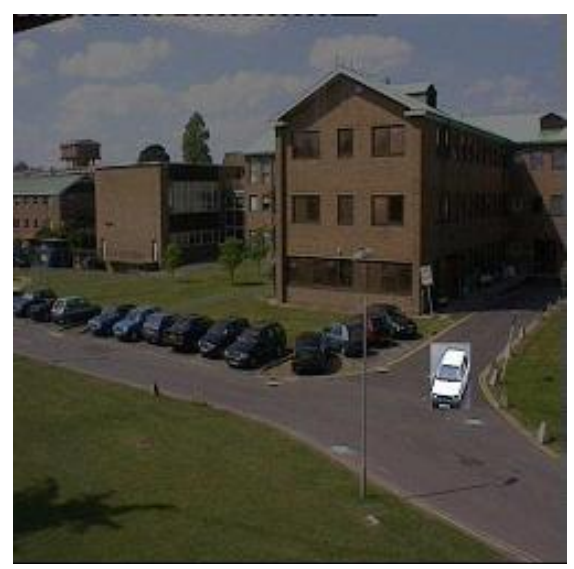

Fig. 3. Testing data 1

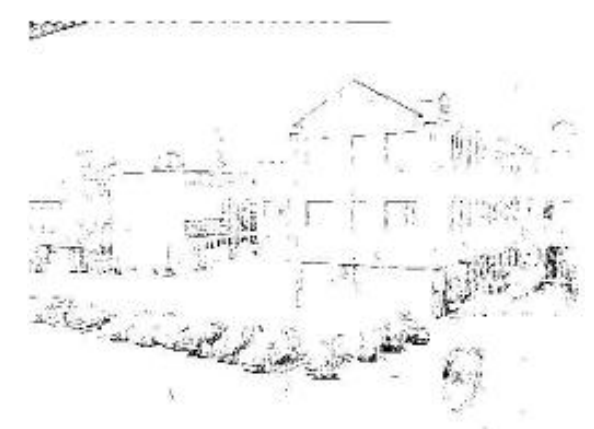

Fig. 4. Output of GMM model

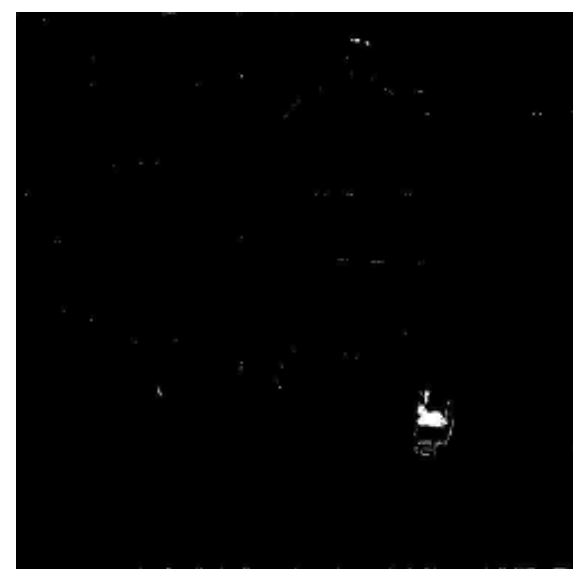

Fig. 5. Output of color distortion model-I

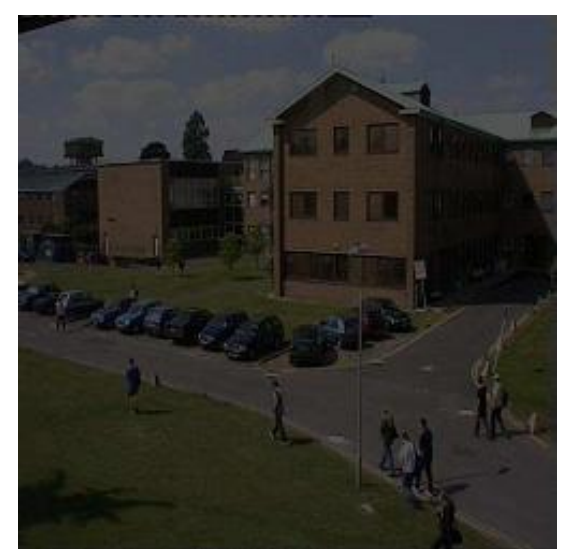

Fig. 6. Testing data set

\section{Background Learning}

In this step background reference image is constructed from the set of training images. Every pixel of background reference e image contain four data i.e. mean color of pixel, mean deviation in color of pixel, generalize background brightness and generalize color distortion of current pixel. Generalize brightness and color distortion is obtained by dividing normal distortion by root mean square value of distortion learnt over set on training images.

\section{Threshold Section}

Now as we have background image, we need threshold value for generalized color distortion which tell us how much color distortion is allowed in the background. Generalized color distortion value over $\mathrm{N}^{*} \mathrm{X} * \mathrm{Y}$ sample, where $\mathrm{N}$ is number of background images, $\mathrm{X}$ is number of column and $\mathrm{Y}$ is number of row in image. From this sample we obtained threshold value either by taking maximum possible color distortion over sample as threshold value otherwise threshold value will be taken as value less than maximum possible distortion which gives exceptable result for training images.

\section{E. Classification of Pixels}

Now for images we can find out generalize color and brightness distortion and if we found color distortion above decided threshold we marked that pixel as foreground otherwise it will be marked as background.

\section{F. Gaussian Mixture Model}

Finding the Moving Object is a commonly used class of techniques for segmenting out objects of interest in a scene for applications such as surveillance. It involves comparing an observed image with an estimate of the image if it contained no objects of interest. The areas of the image plane where there is a significant difference between the observed and estimated images indicate the location of the objects of interest. The name "Finding the Moving Object" comes from the simple technique of subtracting the observed image from the estimated image and thresholding the result to generate the objects of interest.

A Gaussian Mixture Model copes up with multimodal background; hence it is widely used in finding the Moving Object. It calculates each pixel-value from all the sample pixels' mean and variance [6]. GMM is created for each pixel and updated with each new frame. At every new frame some of the Gaussians matches the current value, for them, mean and variance is updated by the running average.

Usually the intensity plot of a pixel is a multimodal plot as shown in the Fig.7. Hence a single Gaussian is unable to capture its multimodal behavior causing the requirement for Gaussian Mixture model. Even the Literature studies shows that Gaussian Mixture Model is more suitable in such kind of system; hence we will use it in our system.

\section{RESULTS}

Approaches described above were implemented on training and testing video sequence provided by PETS2001. Results from first approach are quite appreciable but second approach doesn't show much improvement over GMM 
model. Some of the input and output images are shown in next page.

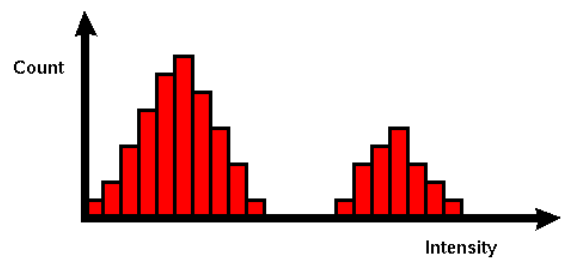

Fig. 7. Intensity plot of a pixel is a multimodal plot

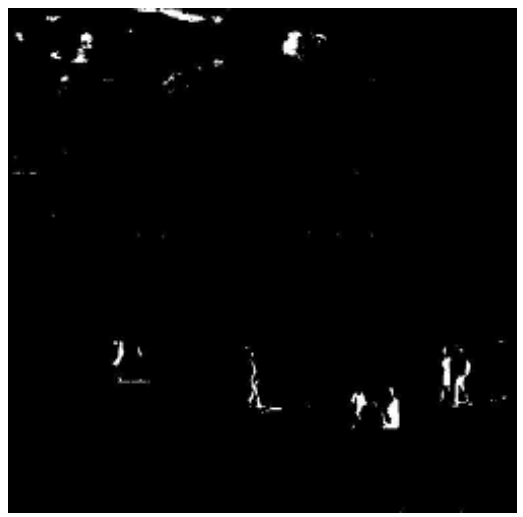

Fig. 8. Output of color and distortion model.

\section{REFERENCES}

[1] S. N. Lim, A. Mittal, L. S. Davis, and N. Paragios, "Fast illumination-invariant background subtraction using two views: error analysis, sensor placement and applications," in Proc. IEEE Computer Society Conference on Computer Vision and Pattern Recognition, vol.1, 2005, pp. 1071-1078.
[2] O. Javed and M. Shah, "Tracking and object classification for automated surveillance," in European Conference on Computer Vision, 2002, pp. 343-357

[3] T. Arat, H. D. Harwood, and L. S. Davis, "A Statistical Approach for Real-time Robust Background Subtraction and Shadow Detection," IEEE International Conference on Computer Vision, vol. 1, pp. 19 (1999)

[4] C. Patel and R. Patel, Gaussian mixture model based Moving object detection from video sequence, the paper will be included in ICWET 2011(Feb) MUMBAI conference proceeding published by ACM digital library. ISBN 978-1-4503-0449-8

[5] P. E. of Tracking and S. PETS, Image dataset, 2001.

[6] M. Piccardi. Background subtraction techniques: A review. April 2003. [Online]. Available: www-staff.it.uts.edu.au/ massimo/ Background Subtraction Review-Piccardi.pdf.

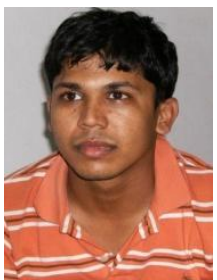

Chirag I. Patel is pursuing Ph.D. in Computer Science \& Engineering from Nirma Institute of Technology, Ahmedabad, Gujarat, India. He received B.E. in Information Technology Engineering from A. D. Patel Institute of Technology, New Vallabh Vidyanagar, Gujarat, India, in 2006, and the M. Tech. in Computer Science \& Engineering from Nirma Institute of Technology , Ahmedabad, Gujarat, India in 2009. His research interests are in video processing, statistical image processing and $3 \mathrm{D}$ vision.

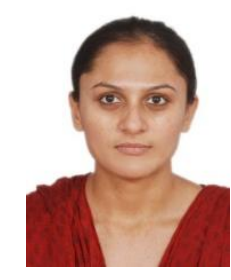

Ripal Patel obtained the B.E. degree in Electronics \& Communication Engineering from A. D. Patel Institute of Technology, New Vallabh Vidyanagar, Gujarat, India, in 2006, and the M.E. in electronics \& communication Engineering from Dharamsinh Desai University, Nadiad, Gujarat, India in 2009. Her research interests are Computer Vision, Texture Classification, Video Processing and Image Registration. 